\title{
Robust Kalman Filtering under Model Perturbations
}

\author{
Mattia Zorzi
}

\begin{abstract}
We consider a family of divergence-based minimax approaches to perform robust filtering. The mismodeling budget, or tolerance, is specified at each time increment of the model. More precisely, all possible model increments belong to a ball which is formed by placing a bound on the Tau-divergence family between the actual and the nominal model increment. Then, the robust filter is obtained by minimizing the mean square error according to the least favorable model in that ball. It turns out that the solution is a family of Kalman like filters. Their gain matrix is updated according to a risk sensitive like iteration where the risk sensitivity parameter is now time varying. As a consequence, we also extend the risk sensitive filter to a family of risk sensitive like filters according to the Tau-divergence family.
\end{abstract}

\section{Index Terms}

Robust Kalman filtering, Tau-divergence family, minimax problem, risk sensitive filtering.

\section{INTRODUCTION}

Kalman filter is ubiquitous in many applications. The main reason is due by its iterative structure, allowing its implementation very simple. On the other hand, this filter is designed with respect to a linear state space model. The latter is often inadequate to describe phenomena, accordingly the resulting Kalman filter does not perform well in the practice. Since the beginning, therefore, it was clear the importance to develop robust versions of the standard Kalman filter.

Robust filtering can be performed according to the risk sensitive approach, [14], [13], [1], [7], [12], [10]. Here, the robust estimator is designed according to the nominal model but in

This work has been partially supported by the FIRB project "Learning meets time" (RBFR12M3AC) funded by MIUR.

M. Zorzi is with the Dipartimento di Ingegneria dell'Informazione, Università degli studi di Padova, via Gradenigo 6/B, 35131 Padova, Italy (zorzimatedei.unipd.it). 
such a way to avoid large errors. The sensitivity to large errors is tuned by the so called risk sensitivity parameter. It is worth noting this approach has been interpreted as a minimax problem [2], [15], [4], [5], [6]. The appealing aspect of the risk sensitive approach is that the solution is a Kaman like filter. On the other hand, the risk sensitive parameter is not explicitly connected to the discrepancy between the actual and the nominal model. Recently, a divergence-based minimax approach has been proposed in [8], [9], [20]. More precisely, in [8] the robust static estimation problem of a signal given noisy observations has been considered. Here, all possible models belong to a ball which is formed by placing a bound on the Kullback-Leibler divergence between the actual and the nominal model. This bound, say tolerance, represents the mismodeling budged. Then, the robust filter is obtained by minimizing the mean square error according to the least favorable model in this ball. It turns out that the Bayes estimator is robust under model uncertainty characterized by this ball. In [9], a dynamic extension to this problem (i.e. a robust filtering problem) has been considered. More precisely, drawing inspiration from [3], [11], the mismodeling budged is specified to each time increment of the model, that is the model uncertainty is expressed in an incremental way. Roughly speaking, the idea is to iterate the Bayes estimator with the least favorable statistics found in [8]. It turns out the robust estimator has a Kalman like structure. More precisely, it is a risk sensitivity like filter, where the risk sensitivity parameter is now time varying.

In [19], the robust static estimation problem proposed in [8] has been extended, in the Gaussian case, to a family of uncertainty classes. The latter is formed by placing a bound on a set of divergences (called $\tau$-divergence family, [17]) between the actual and the nominal model. This particular divergence family is chosen because, in contrast to the alpha and the beta family [18], [16], [21], it allows to characterize uncertainty balls for which the Bayes estimator is still robust.

The contribution of this paper is to extend the robust Kalman filter in [9] to a family of robust Kalman filters parametrized by the $\tau$-divergence family using the results in [19]. This family of filters is characterized by a time varying risk sensitive parameter. Therefore, by adopting the perspective given in [2], we also extend the risk sensitive filter to a family of risk sensitive like filters parametrized by the $\tau$-divergence family, say $\tau$-risk sensitive filters. Finally, we present a simulation study which shows that parameter $\tau$ tunes how conservative the robust filter is.

In the paper we will use the following notation. $\|x\|$ denotes the Euclidean norm of $x \in \mathbb{R}^{n}$. $\|x\|_{A}$ denotes the weighted Euclidean norm with $A$ symmetric and positive definite. 


\section{RobUSt StATIC ESTIMATION}

We review the robust static estimation problem under model uncertainty characterized by the $\tau$-divergence family introduced in [19]. Let $x \in \mathbb{R}^{n}$ and $y \in \mathbb{R}^{p}$ be two jointly Gaussian random vectors. Let $z:=\left[x^{T} y^{T}\right]^{T}$. Its joint nominal probability density $f$ is

$$
f(z)=\frac{1}{\sqrt{(2 \pi)^{p+n} \operatorname{det} K_{z}}} \exp \left(-\frac{1}{2}\left(z-m_{z}\right)^{T} K_{z}^{-1}\left(z-m_{z}\right)\right)
$$

where the mean vector $m_{z} \in \mathbb{R}^{n+p}$ and the covariance matrix $K_{z} \in \mathcal{Q}_{+}^{n+p}$ are known. We conformably partition the mean vector and the covariance matrix of $z$ according to $x$ and $y$ :

$$
m_{z}=\left[\begin{array}{l}
m_{x} \\
m_{y}
\end{array}\right], \quad K_{z}=\left[\begin{array}{cc}
K_{x} & K_{x y} \\
K_{y x} & K_{y}
\end{array}\right] .
$$

Let $\tilde{f}$ denote the actual joint probability density of $z$

$$
\tilde{f}(z)=\frac{1}{\sqrt{(2 \pi)^{p+n} \operatorname{det} \tilde{K}_{z}}} \exp \left(-\frac{1}{2}\left(z-m_{z}\right)^{T} \tilde{K}_{z}^{-1}\left(z-m_{z}\right)\right)
$$

where the mean vector $\tilde{m}_{z} \in \mathbb{R}^{n+p}$ and the covariance matrix $\tilde{K}_{z} \in \mathcal{Q}_{+}^{n+p}$ are unknown. Since both $f$ and $\tilde{f}$ are Gaussian, the deviation between $f$ and $\tilde{f}$ may be directly measured by the deviation between $\left(m_{z}, K_{z}\right)$ and $\left(\tilde{m}_{z}, \tilde{K}_{z}\right)$ through the $\tau$-divergence, [19]:

$$
\begin{aligned}
& \mathcal{D}_{\tau}(\tilde{f} \| f)= \\
& \begin{cases}\left\|\Delta m_{z}\right\|_{K_{z}^{-1}}^{2}+\operatorname{tr}\left(-\log \left(\tilde{K}_{z} K_{z}^{-1}\right)\right. & \tau=0 \\
\left.\quad+\tilde{K}_{z} K_{z}^{-1}-I_{n+p}\right), & \\
\left\|\Delta m_{z}\right\|_{\frac{1}{1-\tau} K_{z}^{-1}}^{2} \operatorname{tr}\left(-\frac{1}{\tau(1-\tau)}\left(L_{z}^{-1} \tilde{K}_{z} L_{z}^{-T}\right)^{\tau}\right. & \\
\left.\quad+\frac{1}{1-\tau} \tilde{K}_{z} K_{z}^{-1}+\frac{1}{\tau} I_{n+p}\right), & 0<\tau<1 \\
\delta_{\infty}\left(\Delta m_{z}\right)+\operatorname{tr}\left(L_{z}^{-1} \tilde{K}_{z} L_{z}^{-T} \log \left(L_{z}^{-1} \tilde{K}_{z} L_{z}^{-T}\right)\right. & \\
\left.-\tilde{K}_{z} K_{z}^{-1}+I_{n+p}\right), & \tau=1\end{cases}
\end{aligned}
$$

where $L_{z}$ is such that $K_{z}=L_{z} L_{z}^{T}, \Delta m_{z}=\tilde{m}_{z}-m_{z}$, and

$$
\delta_{\infty}(v):=\left\{\begin{array}{cc}
0 & \text { if } v=0 \\
\infty & \text { otherwise. }
\end{array}\right.
$$

Note that, $\mathcal{D}_{\tau}(\tilde{f} \| f) \geq 0$ and equality holds if and only if $\tilde{f}=f$. This divergence takes root in the prediction theory. Let $e^{N}=L_{z}^{-1}\left(z-m_{z}\right)$ with $z \sim \tilde{f}$. $e^{N}$ can be understood as a normalized 
prediction error, where $m_{z}$ represents the minimum variance prediction of $z$ based on $f$. If $\tilde{f}=f$, then $e^{N}$ has zero mean and covariance matrix $I$. Hence, this divergence measures the discrepancy between $e^{N}$ and the Gaussian random vector with zero mean and covariance $I$. We consider the closed ball centered on $f$ :

$$
\mathcal{B}_{\tau}:=\left\{f \text { s.t. } \mathcal{D}_{\tau}(\tilde{f} \| f) \leq c\right\}
$$

where $c \in \mathbb{R}_{+}$is a fixed tolerance. Accordingly, $\mathcal{B}_{\tau}$ represents the set of all possible probability densities of $z$ consistent with the allowed mismodelling budget.

The robust estimator of $x$ given $y$ is designed according to the minimax point of view [8], [6]. More precisely, whenever we seek to design an estimator minimizing a suitable loss function, an hostile player, say "nature", conspires to select the worst possible probability density in $\mathcal{B}_{\tau}$. Let $g(y)$ denote an estimator of $x$ based on the observation vector $y$. The optimal robust filter is solution to the following minimax problem

$$
\min _{g \in \mathcal{G}} \max _{\tilde{f} \in \mathcal{B}_{\tau}} J(\tilde{f}, g)
$$

where

$$
J(\tilde{f}, g)=\mathbb{E}_{\tilde{f}}\left[\|x-g(y)\|^{2}\right]=\int_{\mathbb{R}^{n+p}}\|x-g(y)\|^{2} \tilde{f}(z) \mathrm{d} z
$$

denotes the mean square error and $\mathcal{G}$ denotes the set of all estimators $g(y)$ such that $\mathbb{E}_{\tilde{f}}\left[\|g(y)\|^{2}\right]$ is finite for any $\tilde{f} \in \mathcal{B}_{\tau}$.

Theorem 2.1: Let $0 \leq \tau \leq 1$. The least favorable probability density $\tilde{f}^{\circ}$ has mean vector $\tilde{m}_{z}^{\circ}=m_{z}$ and covariance matrix with the following structure

$$
\tilde{K}_{z}^{\circ}=\left[\begin{array}{cc}
\tilde{K}_{x} & K_{x y} \\
K_{y x} & K_{y}
\end{array}\right]
$$

wherein only the covariance of $x$ is perturbed with respect to the nominal covariance matrix. Let

$$
\begin{aligned}
& P=K_{x}-K_{x y} K_{y}^{-1} K_{y x} \\
& V=\tilde{K}_{x}-K_{x y} K_{y}^{-1} K_{y x}
\end{aligned}
$$

denote the nominal and the perturbed a posteriori covariance matrix of $x$ given $y$. Then,

$$
V= \begin{cases}L_{P}\left(I_{n}-\theta(1-\tau) L_{P}^{T} L_{P}\right)^{\frac{1}{\tau-1}} L_{P}^{T}, & 0 \leq \tau<1 \\ L_{P} \exp \left(\theta L_{P}^{T} L_{P}\right) L_{P}^{T}, & \tau=1\end{cases}
$$


where $L_{P}$ is such that $P=L_{P} L_{P}^{T}$. Here $\theta^{-1}$, with $\theta^{-1}>(1-\tau)\|P\|$, is the unique Lagrange multiplier satisfying the relation $c=\gamma_{\tau}(P, \theta)$ where

$$
\begin{aligned}
& \gamma_{\tau}(P, \theta)= \\
& \left\{\begin{array}{lc}
-\log \operatorname{det}\left(I_{n}-\theta P\right)^{-1}+\operatorname{tr}\left(\left(I_{n}-\theta P\right)^{-1}-I_{n}\right), & \tau=0 \\
\operatorname{tr}\left(-\frac{1}{\tau(1-\tau)}\left(I_{n}-\theta(1-\tau) L_{P}^{T} L_{P}\right)^{\frac{\tau}{\tau-1}}\right. & \\
\left.+\frac{1}{1-\tau}\left(I_{n}-\theta(1-\tau) L_{P}^{T} L_{P}\right)^{\frac{1}{\tau-1}}+\frac{1}{\tau} I_{n}\right), & 0<\tau<1 \\
\operatorname{tr}\left(\exp \left(\theta L_{P}^{T} L_{P}\right)\left(\theta L_{P}^{T} L_{P}-I_{n}\right)+I_{n}\right), & \tau=1
\end{array}\right.
\end{aligned}
$$

The optimal robust estimator is the Bayes estimator

$$
g^{\circ}(y)=G^{\circ}\left(y-m_{y}\right)+m_{x}
$$

with $G^{\circ}=K_{x y} K_{y}^{-1}$.

Theorem 2.1 shows that the Bayes estimator is robust with respect to the uncertainty class, parametrized by $\tau$, in (2). Clearly, this optimality holds in the Gaussian case. Without this assumption, the least favorable probability density could be more different than the one in Theorem 2.1.

Corollary 2.1: Let $\theta>0$ be a priori fixed and such that $\theta^{-1}>(1-\tau)\|P\|$. Consider the minimax problem

$$
\min _{g \in \mathcal{G}} \max _{\tilde{f} \in \mathcal{B}_{\tau}} \mathbb{E}_{\tilde{f}}\left[\|x-g(y)\|^{2}\right]-\theta^{-1} \mathcal{D}_{\tau}(\tilde{f} \| f)
$$

where $\mathcal{B}_{\tau}=\left\{\tilde{f}\right.$ s.t. $\left.\mathcal{D}_{\tau}(\tilde{f} \| f)<\infty\right\}$ and $\mathcal{G}$ is the set of all estimators such that $\mathbb{E}_{\tilde{f}}\left[\|g(y)\|^{2}\right]$ is finite for any $\tilde{f} \in \mathcal{B}_{\tau}$. Then, the least favorable probability density $\tilde{f}_{t}^{\circ}$ has mean vector $\tilde{m}_{z}^{\circ}=m_{z}$ and covariance matrix $\tilde{K}_{z}^{\circ}$ as in (4). The perturbed a posteriori covariance matrix $V$ of $x$ given $y$ is in (5). Moreover, its relation with $P$ is given by (6) where $\theta$ now has been a priori chosen. The optimal estimator is the Bayes estimator $(8)$.

\section{ROBUST FILTERING PROBLEM}

We consider a nominal Gauss-Markov state space model of the form

$$
\begin{array}{r}
x_{t+1}=A_{t} x_{t}+B_{t} v_{t} \\
y_{t}=C_{t} x_{t}+D_{t} v_{t}
\end{array}
$$


where $x_{t} \in \mathbb{R}^{n}$ is the state process, $y_{t} \in \mathbb{R}^{p}$ is the observation process, and $v_{t} \in \mathbb{R}^{m}$ is WGN with unit variance, i.e. $\mathbb{E}\left[v_{t} v_{s}^{T}\right]=I_{m} \delta_{t-s}$ where $\delta_{t}$ denotes the Kronecker delta function. We assume that the noise $v_{t}$ is independent of the initial state, whose nominal distribution is given by $f_{0}\left(x_{0}\right) \sim \mathcal{N}\left(\hat{x}_{0}, V_{0}\right)$. Let $z_{t}=\left[x_{t+1}^{T} y_{t}^{T}\right]^{T}$. Model $[9$ is characterized by the nominal transition probability density of $z_{t}$ given $x_{t}$ :

$$
\phi_{t}\left(z_{t} \mid x_{t}\right) \sim \mathcal{N}\left(\left[\begin{array}{l}
A_{t} \\
C_{t}
\end{array}\right] x_{t},\left[\begin{array}{c}
B_{t} \\
D_{t}
\end{array}\right]\left[\begin{array}{ll}
B_{t}^{T} & D_{t}^{T}
\end{array}\right]\right) .
$$

As noticed in [9], when entropy-like indexes are used to measure the proximity of statistical models, all the relations between dynamic variables or observations should be uncertain, otherwise those indexes take infinite value. To avoid such a situation, we assume that the noise $v_{t}$ affects all the components of the dynamics and observations in (9), possibly with a very small variance for relations which are viewed as almost certain. Therefore, the covariance matrix

$$
K_{z_{t} \mid x_{t}}=\left[\begin{array}{c}
B_{t} \\
D_{t}
\end{array}\right]\left[\begin{array}{cc}
B_{t}^{T} & D_{t}^{T}
\end{array}\right]
$$

is positive definite. Moreover, the matrix $\Gamma_{t}=\left[B_{t}^{T} D_{t}^{T}\right]^{T}$ has full column rank, and without loss of generality we can assume $\Gamma_{t}$ is square and invertible, so that $m=n+p$. Otherwise, we can compress the column space of $\Gamma_{t}$ and remove noise components which do not affect model (9).

We adopt the minimax approach proposed [9, Section III] to characterize the robust filter. Let $\tilde{\phi}_{s}\left(z_{t} \mid x_{t}\right)$ be the least favorable transition probability density of $z_{t}$ given $x_{t}$. Let $\tilde{f}_{t}\left(x_{t} \mid Y_{t-1}\right)$ be the a priori probability density of $x_{t}$ conditioned on the observations $Y_{t-1}=\left\{y_{s}, \quad 0 \leq s \leq t-1\right\}$ and based on the least favorable model. We introduce the marginal probability densities

$$
\begin{aligned}
& \bar{f}_{t}\left(z_{t} \mid Y_{t-1}\right)=\int \phi_{t}\left(z_{t} \mid x_{t}\right) \tilde{f}_{t}\left(x_{t} \mid Y_{t-1}\right) \mathrm{d} x_{t} \\
& \tilde{f}_{t}\left(z_{t} \mid Y_{t-1}\right)=\int \tilde{\phi}_{t}\left(z_{t} \mid x_{t}\right) \tilde{f}_{t}\left(x_{t} \mid Y_{t-1}\right) \mathrm{d} x_{t} .
\end{aligned}
$$

Note that, $\bar{f}_{t}\left(z_{t} \mid Y_{t-1}\right)$ can be viewed as the pseudo-nominal density of $z_{t}$ conditioned on $Y_{t-1}$ computed from the conditional least favorable density $\tilde{f}_{t}\left(x_{t} \mid Y_{t-1}\right)$ and the nominal transition probability density $\phi_{t}\left(x_{t} \mid z_{t}\right)$. As in [9], we assume that

$$
\tilde{f}_{t}\left(x_{t} \mid Y_{t-1}\right) \sim \mathcal{N}\left(\hat{x}_{t}, V_{t}\right)
$$


In this way the conditional probability density $\bar{f}_{t}\left(z_{t} \mid Y_{t-1}\right)$ is Gaussian. We make the additional assumption that $\tilde{\phi}\left(z_{t} \mid x_{t}\right)$ is such that $\tilde{f}_{t}\left(z_{t} \mid Y_{t-1}\right)$ is Gaussian. In [9], the latter assumption was not made. However, it is worth noting that the least favorable solution found is such that (12) is Gaussian, see Remark 3.2. Therefore, we can measure the deviance between $\tilde{\phi}_{t}$ and $\phi_{t}$ as deviance between $\tilde{f}_{t}\left(z_{t} \mid Y_{t-1}\right)$ and $\bar{f}_{t}\left(z_{t} \mid Y_{t-1}\right)$ using the $\tau$-divergence 11 . Then, we assume that $\tilde{\phi}_{t}$ belongs to the closed ball about $\phi_{t}: \mathcal{B}_{t, \tau}=\left\{\tilde{\phi}_{t}\left(z_{t} \mid x_{t}\right)\right.$ s.t. $\left.\mathcal{D}_{\tau}\left(\tilde{f}_{t} \| \bar{f}_{t}\right) \leq c_{t}\right\}$ where $c_{t} \in \mathbb{R}_{+}$ is the tolerance specified at each time step. Let $\mathcal{G}_{t}$ denote the class of estimators with finite second-order moments with respect all densities $\tilde{\phi}_{t}\left(z_{t} \mid x_{t}\right) \tilde{f}_{t}\left(x_{t} \mid Y_{t-1}\right)$ such that $\tilde{\phi}_{t}\left(z_{t} \mid x_{t}\right) \in \mathcal{B}_{t, \tau}$. The the robust filter is characterized by the following minimax problem

$$
\left(g_{t}^{\circ}, \tilde{\phi}_{t}^{\circ}\right)=\arg \min _{g_{t} \in \mathcal{G}_{t} \tilde{\phi}_{t} \in \mathcal{B}_{t, \tau}} J_{t}\left(\tilde{\phi}_{t}, g_{t}\right)
$$

where

$$
\begin{aligned}
J_{t} & \left(\tilde{\phi}_{t}, g_{t}\right)=\mathbb{E}_{\tilde{f}_{t}}\left[\left\|x_{t+1}-g_{t}\left(y_{t}\right)\right\|^{2} \mid Y_{t-1}\right] \\
& =\iint\left\|x_{t+1}-g_{t}\left(y_{t}\right)\right\|^{2} \tilde{\phi}_{t}\left(z_{t} \mid x_{t}\right) \tilde{f}_{t}\left(x_{t} \mid Y_{t-1}\right) \mathrm{d} x_{t} \mathrm{~d} z_{t}
\end{aligned}
$$

denotes the mean square error of the estimator $\hat{x}_{t+1}=g_{t}^{\circ}\left(y_{t}\right)$ of $x_{t+1}$ evaluated with respect to the transition density $\tilde{\phi}_{t}$ in $\mathcal{B}_{t, \tau}$. It is worth noting that $\hat{x}_{t+1}$ depends on $Y_{t}$, and not only on $y_{t}$, but this dependency is suppressed to simplify the notations.

Remark 3.1: In the minimax problem (14) we require that $\tilde{f}_{t}\left(z_{t} \mid Y_{t-1}\right)$ defined in 12$)$ is a conditional probability density, that is

$$
\iint \tilde{\phi}_{t}\left(z_{t} \mid x_{t}\right) \tilde{f}_{t}\left(x_{t} \mid Y_{t-1}\right) \mathrm{d} z_{t} \mathrm{~d} x_{t}=1,
$$

but we do not require that $\tilde{\phi}_{t}\left(z_{t} \mid x_{t}\right)$ is a transition probability density for each $x_{t}$. Therefore, the a priori conditional probability density $\tilde{f}_{t}\left(x_{t} \mid Y_{t-1}\right)$ is not required to coincide with the $a$ posteriori one computed from $\tilde{\phi}_{t}\left(z_{t} \mid x_{t}\right) \tilde{f}_{t}\left(x_{t} \mid Y_{t-1}\right)$.

Remark 3.2: In [9], $\tilde{\phi}_{t}\left(z_{t} \mid x_{t}\right)$ is not required to be such that 12 is Gaussian. The constraint on $\tilde{\phi}_{t}\left(z_{t} \mid x_{t}\right)$ is that $\mathbb{D}_{K L}\left(\tilde{f}_{t} \| \bar{f}_{t}\right) \leq c_{t}$ where $\mathbb{D}_{K L}$ is the Kullback-Leibler divergence among probability densities. On the other hand, the solution $\tilde{\phi}_{t}^{\circ}\left(z_{t} \mid x_{t}\right)$ to the corresponding minimax problem is such that 12 is Gaussian, see [9, Formula (16)]. Hence, the corresponding $\tilde{f}_{t}^{\circ}\left(z_{t} \mid Y_{t-1}\right)$ is Gaussian. Note that, $\mathrm{D}_{K L}\left(\tilde{f}_{t} \| \bar{f}_{t}\right)=\mathcal{D}_{0}\left(\tilde{f}_{t} \| \bar{f}_{t}\right)$ when $\tilde{f}_{t}, \bar{f}_{t}$ are Gaussian. We conclude that, for $\tau=0$, the solution to (14) coincides with the one in [9]. 


\section{Robust Kalman Filters}

We show that the optimal robust estimator solution to the minimax problem (14) is a Kalman like filter parametrized by $\tau$. In this way, we obtain a parametric family of robust Kalman filters. First, Problem (14) can be reformulated as the static minimax problem (3). Consider the ball $\overline{\mathcal{B}}_{t, \tau}=\left\{\tilde{f}_{t}\left(z_{t} \mid Y_{t-1}\right)\right.$ s.t. $\left.\mathcal{D}_{\tau}\left(\tilde{f}_{t} \| \bar{f}_{t}\right) \leq c_{t}\right\}$ which is the set of all probability densities having structure 12 with $\tilde{\phi}_{t} \in \mathcal{B}_{t, \tau}$. The equivalent minimax problem is

$$
\left(\tilde{f}_{t}^{\circ}, g_{t}^{\circ}\right)=\arg \min _{g_{t} \in \mathcal{G}_{t}} \max _{\tilde{f}_{t} \in \overline{\mathcal{B}}_{t, \tau}} \bar{J}_{t}\left(\tilde{f}_{t}, g_{t}\right)
$$

where

$$
\bar{J}_{t}\left(\tilde{f}_{t}, g_{t}\right)=\int\left\|x_{t+1}-g_{t}\left(y_{t}\right)\right\|^{2} \tilde{f}_{t}\left(z_{t} \mid Y_{t-1}\right) \mathrm{d} z_{t} .
$$

In view of $(10)$ and $(13)$, the pseudo-nominal density is Gaussian

$$
\bar{f}_{t}\left(z_{t} \mid Y_{t-1}\right) \sim \mathcal{N}\left(\left[\begin{array}{c}
A_{t} \\
C_{t}
\end{array}\right] \hat{x}_{t}, K_{z_{t}}\right)
$$

where the conditional covariance matrix $K_{z_{t}}$ is given by

$$
\begin{aligned}
K_{z_{t}} & =\left[\begin{array}{cc}
K_{x_{t+1}} & K_{x_{t+1}, y_{t}} \\
K_{y_{t}, x_{t+1}} & K_{y_{t}}
\end{array}\right] \\
& =\left[\begin{array}{c}
A_{t} \\
C_{t}
\end{array}\right] V_{t}\left[\begin{array}{ll}
A_{t}^{T} & C_{t}^{T}
\end{array}\right]+\left[\begin{array}{c}
B_{t} \\
D_{t}
\end{array}\right]\left[\begin{array}{cc}
B_{t}^{T} & D_{t}^{T}
\end{array}\right] .
\end{aligned}
$$

Applying Theorem 2.1 with $f \mapsto \bar{f}_{t}, \tilde{f} \mapsto \tilde{f}_{t}$ and $g \mapsto g_{t}$, the least favorable conditional density $\tilde{f}_{t}^{\circ}\left(z_{t} \mid Y_{t-1}\right)$ is such that

$$
\tilde{f}_{t}^{\circ}\left(z_{t} \mid Y_{t-1}\right) \sim \mathcal{N}\left(\left[\begin{array}{c}
A_{t} \\
C_{t}
\end{array}\right] \hat{x}_{t}, \tilde{K}_{z_{t}}^{\circ}\right)
$$

where the least favorable conditional covariance matrix is

$$
\tilde{K}_{z t}^{\circ}=\left[\begin{array}{cc}
\tilde{K}_{x_{t+1}} & K_{x_{x+1} y_{t}} \\
K_{y_{t} x_{t+1}} & K_{y_{t}}
\end{array}\right] .
$$

Let

$$
\begin{aligned}
& P_{t+1}=K_{x_{t+1}}-K_{x_{t+1}, y_{t}} K_{y_{t}}^{-1} K_{y_{t}, x_{t+1}} \\
& V_{t+1}=\tilde{K}_{x_{t+1}}-K_{x_{t+1}, y_{t}} K_{y_{t}}^{-1} K_{y_{t}, x_{t+1}}
\end{aligned}
$$


denote the nominal and the least favorable conditional covariance of $x_{x+1}$ given $Y_{t}$. Then,

$$
\begin{aligned}
& V_{t+1}= \\
& \begin{cases}L_{P_{t+1}}\left(I_{n}-\theta_{t}(1-\tau) L_{P_{t+1}}^{T} L_{P_{t+1}}\right)^{\frac{1}{\tau-1}} L_{P_{t+1}}^{T}, & 0<\tau<1 \\
L_{P_{t+1}} \exp \left(\theta_{t} L_{P_{t+1}}^{T} L_{P_{t+1}}\right) L_{P_{t+1}}^{T}, & \tau=1\end{cases}
\end{aligned}
$$

where $L_{P_{t+1}}$ is such that $P_{t+1}=L_{P_{t+1}} L_{P_{t+1}}^{T}$ and $\theta_{t}^{-1}>(1-\tau)\left\|P_{t+1}\right\|$ is the unique solution to $c_{t}=\gamma_{\tau}\left(P_{t+1}, \theta_{t}\right)$ where $\gamma_{\tau}$ has been defined in (7). The optimal robust estimator takes the form,

$$
\hat{x}_{t+1}=g_{t}^{\circ}\left(y_{t}\right)=A_{t} \hat{x}_{t}+G_{t}\left(y_{t}-C_{t} \hat{x}_{t}\right)
$$

with gain matrix $G_{t}=K_{x_{t+1}, y_{t}} K_{y_{t}}^{-1}$. From $[17$, we obtain

$$
\begin{aligned}
G_{t} & =\left(A_{t} V_{t} C_{t}^{T}+B_{t} D_{t}^{T}\right)\left(C_{t} V_{t} C_{t}^{T}+D_{t} D_{t}^{T}\right)^{-1} \\
P_{t+1} & =A_{t} V_{t} A_{t}^{T}-G_{t}\left(C_{t} V_{t} C_{t}^{T}+D_{t} D_{t}^{T}\right) G_{t}^{T}+B_{t} B_{t}^{T} .
\end{aligned}
$$

Algorithm 1 shows the iterative scheme of the optimal robust estimator we found for the case $0 \leq \tau<1$. The algorithm for the limit case $\tau=1$ is the same with the exception that the updating of $V_{t+1}$ is different. It is clear that the robust filter has the same iterative structure of the Kalman filter with the exception that $P_{t}$ is applied a distortion through matrix $V_{t}$. In particular, $G_{t}$ is governed by a Riccati-like equation.

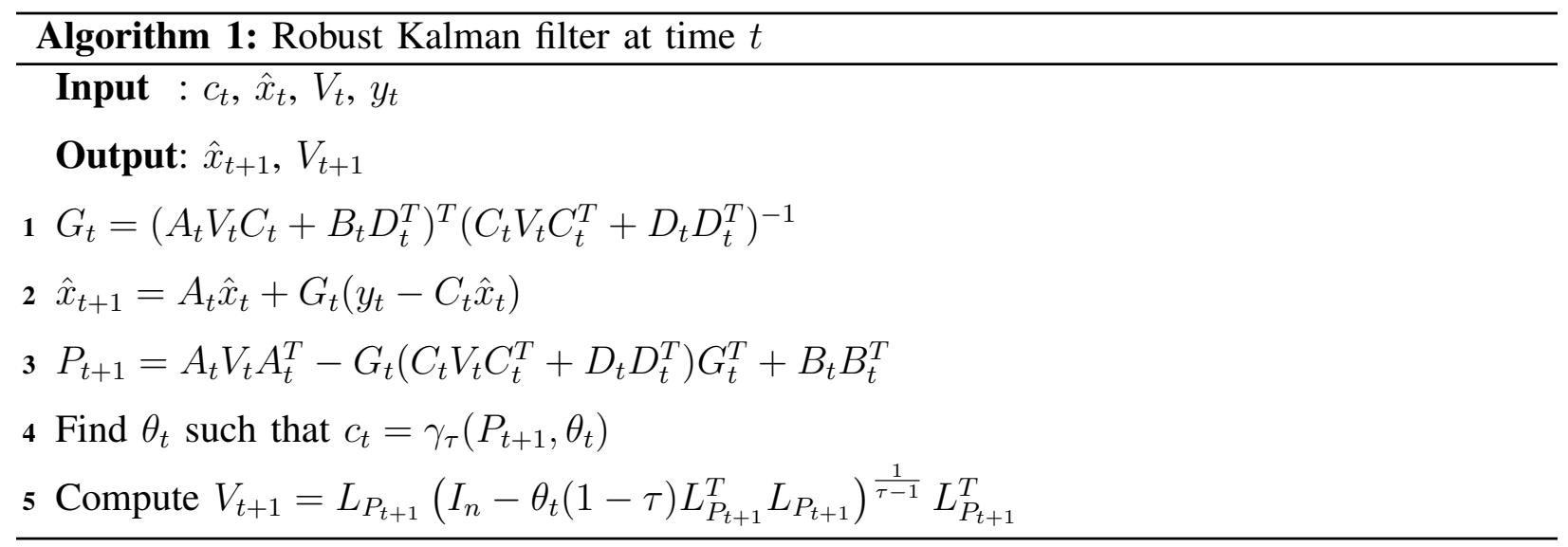

It remains to characterize the least favorable transition density $\tilde{\phi}_{t}^{\circ}\left(z_{t} \mid x_{t}\right)$. It is not difficult to 
prove that, [19, Theorem 2.1],

Accordingly

$$
\begin{gathered}
K_{z_{t}}^{-1}=\left[\begin{array}{cc}
I_{n} & 0 \\
-G_{t}^{T} & I_{p}
\end{array}\right]\left[\begin{array}{cc}
P_{t+1}^{-1} & 0 \\
0 & K_{y_{t}}^{-1}
\end{array}\right]\left[\begin{array}{cc}
I_{n} & -G_{t} \\
0 & I_{p}
\end{array}\right] \\
\left(\tilde{K}_{z_{t}}^{\circ}\right)^{-1}=\left[\begin{array}{cc}
I_{n} & 0 \\
-G_{t}^{T} & I_{p}
\end{array}\right]\left[\begin{array}{cc}
V_{t+1}^{-1} & 0 \\
0 & K_{y_{t}}^{-1}
\end{array}\right]\left[\begin{array}{cc}
I_{n} & -G_{t} \\
0 & I_{p}
\end{array}\right] .
\end{gathered}
$$

$$
K_{z_{t}}^{-1}-\left(\tilde{K}_{z_{t}}^{\circ}\right)^{-1}=\left[\begin{array}{c}
I_{n} \\
-G_{t}^{T}
\end{array}\right] \Phi_{t}\left[\begin{array}{ll}
I_{n} & -G_{t}
\end{array}\right]
$$

where $\Phi_{t}=P_{t+1}^{-1}-V_{t+1}^{-1}$ which is positive definite. Let $e_{t}=x_{t}-\hat{x}_{t}$ denote the estimation error. Define

$$
\hat{m}_{z_{t}}=\mathbb{E}_{f_{t}}\left[z_{t} \mid Y_{t-1}\right]=\mathbb{E}_{\tilde{f}_{t}^{\circ}}\left[z_{t} \mid Y_{t-1}\right]=\left[\begin{array}{cc}
A_{t}^{T} & C_{t}^{T}
\end{array}\right]^{T} \hat{x}_{t}
$$

Therefore,

$$
\begin{aligned}
\left(z_{t}\right. & \left.-\hat{m}_{z_{t}}\right)^{T}\left(K_{z_{t}}^{-1}-\left(\tilde{K}_{z_{t}}^{\circ}\right)^{-1}\right)\left(z_{t}-\hat{m}_{z_{t}}\right) \\
& =\left(z_{t}-\hat{m}_{z_{t}}\right)^{T}\left[\begin{array}{c}
I_{n} \\
-G_{t}^{T}
\end{array}\right] \Phi_{t}\left[\begin{array}{ll}
I_{n} & -G_{t}
\end{array}\right]\left(z_{t}-\hat{m}_{z_{t}}\right) \\
& =\left\|x_{t+1}-\left(A_{t} \hat{x}_{t}+G_{t}\left(y_{t}-C_{t} \hat{x}_{t}\right)\right)\right\|_{\Phi_{t}}^{2} \\
& =\left\|x_{t+1}-\hat{x}_{t+1}\right\|_{\Phi_{t}}^{2}=\left\|e_{t+1}\right\|_{\Phi_{t}}^{2} .
\end{aligned}
$$

By (18) and (16), we have

$$
\tilde{f}_{t}^{\circ}\left(z_{t} \mid Y_{t-1}\right) \sim \exp \left(\frac{1}{2}\left\|e_{t+1}\right\|_{\Phi_{t}}^{2}\right) \bar{f}_{t}\left(z_{t} \mid Y_{t-1}\right) .
$$

By (11), we obtain

$$
\tilde{f}_{t}^{\circ}\left(z_{t} \mid Y_{t-1}\right) \sim \int \exp \left(\frac{1}{2}\left\|e_{t+1}\right\|_{\Phi_{t}}^{2}\right) \phi_{t}\left(z_{t} \mid x_{t}\right) \tilde{f}_{t}\left(x_{t} \mid Y_{t-1}\right) \mathrm{d} x_{t} .
$$

and by (12) we conclude that

$$
\tilde{\phi}_{t}^{\circ}\left(z_{t} \mid x_{t}\right)=\frac{1}{M_{t}\left(\Phi_{t}\right)} \exp \left(\frac{1}{2}\left\|e_{t+1}\right\|_{\Phi_{t}}^{2}\right) \phi_{t}\left(z_{t} \mid x_{t}\right)
$$

where the normalizing constant $M_{t}\left(\Phi_{t}\right)$ is such that 15 holds. It is worth noting that in the case $\tau=0$, i.e. the case considered in [9], the distortion is a radial function of the estimation error $e_{t+1}$, because $\Phi_{t}=\theta_{t}^{-1} I_{n}$ for $\tau=0$. On the contrary, in the case $\tau \neq 0$ such distortion is nonradial. 


\section{LEAST-FAVORABLE MOdeL}

For simulation and performance evaluation purposes, in particular for choosing parameters $c_{t}$ and $\tau$, it is important to characterize the least favorable model which is the solution to (14). The idea is to characterize it through (21). Note that, there is a one to one correspondence between $z_{t}$ and $v_{t}$, given $x_{t}$, through the relation

$$
v_{t}=\Gamma_{t}^{-1}\left(z_{t}-\left[\begin{array}{c}
A_{t} \\
C_{t}
\end{array}\right] x_{t}\right)
$$

because matrix $\Gamma_{t}$ is invertible. Accordingly, we can characterize the least favorable model with model (9) where the distortion has been moved now in noise $v_{t}$. Applying the same arguments used in [9], see also [6, Section 17.7], it is not difficult to prove that the least favorable probability density of $v_{t}$ depends on $e_{t}$ and is distributed as $\tilde{\psi}_{t}\left(v_{t} \mid e_{t}\right) \sim \mathcal{N}\left(H_{t} e_{t}, \tilde{K}_{v_{t}}\right)$ where $\tilde{K}_{v_{t}}=\left(I_{n+p}-\right.$ $\left.\left(B_{t}-G_{t} D_{t}\right)^{T}\left(\Omega_{t+1}^{-1}+\Phi_{t}\right)\left(B_{t}-G_{t} D_{t}\right)\right)^{-1}$ and $H_{t}=\tilde{K}_{v_{t}}\left(B_{t}-G_{t} D_{t}\right)^{T}\left(\Omega_{t+1}^{-1}+\Phi_{t}\right)\left(A_{t}-G_{t} C_{t}\right)$. Matrix $\Omega_{t+1}^{-1}$ is computed from the backward recursion

$$
\Omega_{t}^{-1}=\left(A_{t}-G_{t} C_{t}\right)^{T}\left(\Omega_{t+1}^{-1}+\Phi_{t}\right)\left(A_{t}-G_{t} C_{t}\right)+H_{t}^{T} \tilde{K}_{v_{t}}^{-1} H_{t}
$$

where the final point can be initialized with $\Omega_{T+1}^{-1}=0$ and $T$ is the simulation horizon. The backward recursion is due by the fact that integrating $\tilde{\phi}_{t}^{\circ}\left(z_{t} \mid x_{t}\right)$ over $z_{t}$ we obtain a positive function of $e_{t}$, therefore the "nature" has the opportunity to change retroactively the least favorable density of $x_{t}$. It is not difficult to see that the least favorable model admits a state space representation with matrices

$$
\begin{aligned}
& \tilde{A}_{t}=\left[\begin{array}{cc}
A_{t} & B_{t} H_{t} \\
0 & A_{t}-G_{t} C_{t}+\left(B_{t}-G_{t} D_{t}\right) H_{t}
\end{array}\right] \\
& \tilde{B}_{t}=\left[\begin{array}{c}
B_{t} \\
B_{t}-G_{t} D_{t}
\end{array}\right] L_{t}, \tilde{C}_{t}=\left[\begin{array}{ll}
C_{t} & D_{t} H_{t}
\end{array}\right], \tilde{D}_{t}=D_{t} L_{t}
\end{aligned}
$$

and the input is WGN with unit variance. Note that, to construct the least favorable model, first we generate the gains $G_{t}$ performing a forward sweep of the robust filter (19)-20 over interval $[0, T]$, then we generate the matrices $\Omega_{t}$ through a backward sweep over interval $[0, T]$. Therefore, increasing the simulation interval beyond $[0, T]$ requires performing a new backward sweep of recursion (22). Then, we can evaluate the performance of an arbitrary estimator

$$
\hat{x}_{t+1}^{\prime}=A_{t} \hat{x}_{t}^{\prime}+G_{t}^{\prime}\left(y_{t}-C_{t} \hat{x}_{t}^{\prime}\right)
$$


applied to the the least favorable model. Let

$$
\Pi_{t}=\mathbb{E}\left[\left[\begin{array}{l}
e_{t}^{\prime} \\
e_{t}
\end{array}\right]\left[\left(e_{t}^{\prime}\right)^{T} e_{t}^{T}\right]\right]
$$

where $e_{t}$ is the estimation error of the optimal filter $(19)$ and $e_{t}^{\prime}$ is the estimation error of filter (24). Then, it can be proven that $\Pi_{t}$ obeys to the Lyapunov equation, [9],

$$
\begin{aligned}
\Pi_{t+1}= & \left(\tilde{A}_{t}-\left[\begin{array}{c}
G_{t}^{\prime} \\
0
\end{array}\right] \tilde{C}_{t}\right) \Pi_{t}\left(\tilde{A}_{t}-\left[\begin{array}{c}
G_{t}^{\prime} \\
0
\end{array}\right] \tilde{C}_{t}\right)^{T} \\
& +\left(\tilde{B}_{t}-\left[\begin{array}{c}
G_{t}^{\prime} \\
0
\end{array}\right] \tilde{D}_{t}\right)\left(\tilde{B}_{t}-\left[\begin{array}{c}
G_{t}^{\prime} \\
0
\end{array}\right] \tilde{D}_{t}\right)^{T}
\end{aligned}
$$

where $\Pi_{0}=I_{2} \otimes V_{0}$.

\section{Risk SENSITIVE FILTERING}

Consider the robust Kalman filter we presented in Section IV with $\tau=0$. If we replace $\theta_{t}$ with a constant value $\theta$ we recognize immediately that we obtain the risk sensitive filter, [13], [1], [14]. This suggest us that the risk sensitive filter can extended using the $\tau$-divergence family. Consider the Markov-Gauss state space model $(9)$. Let $\bar{f}_{t}\left(z_{t} \mid Y_{t-1}\right)$ be the conditional density of $z_{t}$ given $Y_{t-1}$ based on the model (9) and defined in (16). The classic risk sensitive estimator $g_{t}^{\circ}$ at time $t$ is defined as

$$
g_{t}^{\circ}=\underset{g_{t} \in \mathcal{G}_{t}}{\operatorname{argmin}} \mathbb{E}_{\bar{f}_{t}}\left[\exp \left(\theta\left\|x_{t+1}-g_{t}\left(y_{t}\right)\right\|^{2}\right) \mid Y_{t-1}\right]
$$

where $\mathcal{G}_{t}$ is the set of estimators for which the objective function in 26 is finite. $\theta>0$ is the risk sensitivity parameter. More precisely, the larger $\theta$ is the more the objective function in (26) penalizes estimators with large errors. In [2], it has been shown that the risk sensitive estimator is solution to the following minimax problem

$$
\begin{aligned}
g_{t}^{\circ}=\underset{g_{t} \in \mathcal{G}_{t}}{\operatorname{argminmax}} \tilde{f}_{t} \in \mathcal{B}_{t} & \mathbb{E}_{\tilde{f}_{t}} \\
{[} & \left.\left\|x_{t+1}-g_{t}\left(y_{t}\right)\right\|^{2} \mid Y_{t-1}\right] \\
& -\theta^{-1} \mathbb{D}_{K L}\left(\tilde{f}_{t} \| f_{t}\right)
\end{aligned}
$$

where $\mathcal{B}_{t}=\left\{\tilde{f}_{t}\right.$ s.t. $\left.\mathbb{D}_{K L}\left(\tilde{f}_{t} \| f_{t}\right)<\infty\right\}$. The second term in the objective function in 27) is always nonpositive because $\mathbb{D}_{K L}\left(\tilde{f}_{t} \| f_{t}\right) \geq 0$. For small values of $\theta$, it takes large negative values for conditional densities not close to the nominal one. Therefore, the maximizer is obliged 
to choose a conditional density close to the nominal one. On the contrary, for large values of $\theta$, it takes (negative) values close to zero for some conditional densities not close to the nominal one. In such a situation, the maximizer has the possibility to choose those conditional densities. Note that, this behaviour does not change if we replace $\mathbb{D}_{K L}$ with another divergence measure.

In our setting $\bar{f}_{t}\left(z_{t} \mid Y_{t-1}\right)$ is Gaussian by assumption. In addition, if we assume that $\tilde{f}_{t}\left(z_{t} \mid Y_{t-1}\right)$ is Gaussian, then $\mathbb{D}_{K L}\left(\tilde{f}_{t} \| f_{t}\right)=\mathcal{D}_{0}\left(\tilde{f}_{t} \| f_{t}\right)$ where $\mathcal{D}_{0}$ has been defined in $(1)$. It is then natural to extend the minimax problem (27) to the $\tau$-divergence family:

$$
\begin{gathered}
g_{t}^{\circ}=\underset{g_{t} \in \mathcal{G}_{t}}{\operatorname{argminmax}} \tilde{f}_{t} \in \mathcal{B}_{t} \\
\mathbb{E}_{\tilde{f}_{t}}
\end{gathered}
$$

By applying Corollary 2.1, the optimal $\tau$-risk sensitive estimator takes the form of (19)- 20 ) where

$$
\begin{aligned}
& V_{t+1}= \\
& \begin{cases}L_{P_{t+1}}\left(I_{n}-\theta(1-\tau) L_{P_{t+1}}^{T} L_{P_{t+1}}\right)^{\frac{1}{\tau-1}} L_{P_{t+1}}^{T}, & 0<\tau<1 \\
L_{P_{t+1}} \exp \left(\theta L_{P_{t+1}}^{T} L_{P_{t+1}}\right) L_{P_{t+1}}^{T}, & \tau=1 .\end{cases}
\end{aligned}
$$

It is worth noting that, for the case $0 \leq \tau<1, V_{t+1}$ is defined provided that $0<P_{t+1}<$ $(\theta(1-\tau))^{-1} I_{n}$, while for the case $\tau=1$, it is well defined whenever $P_{t+1}$ is positive definite. The algorithmic scheme is similar to Algorithm 1: the unique difference is that Step 4 is now removed. Finally, while the risk sensitivity parameter of the robust filter of Section $[\mathrm{IV}$ is time varying, and its evolution is governed by $c_{t}$, now it is constant.

\section{Simulation Results}

We consider the time-invariant model (9) with

$$
\begin{array}{rr}
A=\left[\begin{array}{cc}
0.1 & 1 \\
0 & 1.2
\end{array}\right], & B=\left[\begin{array}{ccc}
0.01 & 0 & 0 \\
0 & 0.01 & 0
\end{array}\right] \\
C=\left[\begin{array}{cc}
1 & -1
\end{array}\right], & D=\left[\begin{array}{lll}
0 & 0 & 0.1
\end{array}\right]
\end{array}
$$

and $x_{0} \sim \mathcal{N}\left(0, V_{0}\right)$ with $V_{0}=0.01 \cdot I_{2}$. We consider the following three filters: KF is the standard Kalman filter; $\mathrm{RKF}_{0}$ is the robust Kalman filter of Section IV] with $\tau=0$; $\mathrm{RKF}_{1}$ is the robust Kalman filter of Section IV with $\tau=1$. In Figure 1 we show the evolution of the risk sensitivity 
parameter of $\mathrm{RKF}_{0}$ and $\mathrm{RKF}_{1}$ for $c=10^{-1}$. We notice the $\theta_{t}$ is constant in the steady state, that is $\mathrm{RKF}_{0}$ and $\mathrm{RKF}_{1}$ coincide with the risk sensitive filters of Section $\mathrm{VI}$ with $\tau=0, \theta \approx 0.19$ and $\tau=1, \theta \approx 0.23$, respectively, in the steady state.

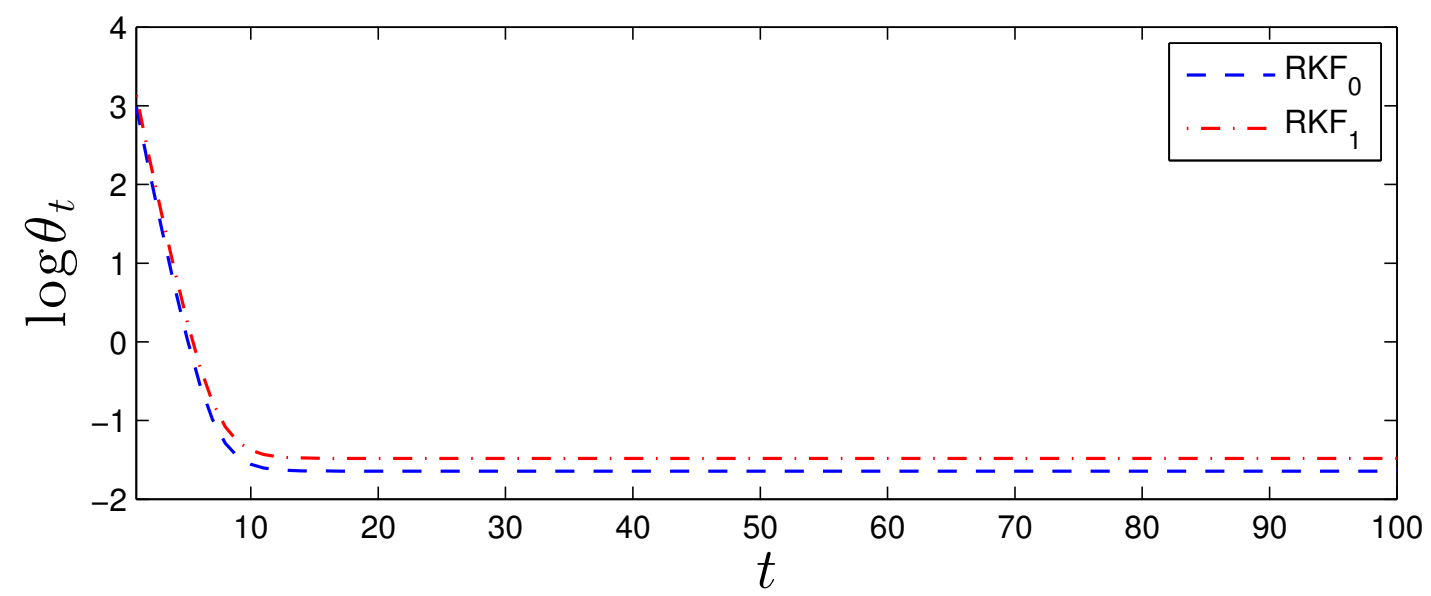

Fig. 1. Evolution of the risk sensitive parameter $\theta_{t}$ with $c=10^{-1}$.

In what follows we evaluate the performance of $\mathrm{RK}, \mathrm{RKF}_{0}$ and $\mathrm{RKF}_{1}$, which have structure (24), applied to the least favorable model (23). More precisely, for each filter, applied to (23), we consider the estimation error $e_{t}^{\prime}=\left[e_{t}^{1} e_{t}^{2}\right]^{T}$. Then, we compute the variance of $e_{t}^{1}$ and $e_{t}^{2}$ through (25). We consider two situations: $c$ large, i.e. nominal and least favorable model are very different; $c$ small, i.e. nominal and least favorable model are similar.

\section{A. Large tolerance}

Here $\mathrm{RKF}_{0}$ and $\mathrm{RKF}_{1}$ have tolerance $c=10^{-1}$. In the first experiment, we apply these filters to the nominal model 9 . The variance of $e_{t}^{1}$ and $e_{t}^{2}$ are depicted in the first row of Figure 2, As expected, KF performs better than the others. Moreover, the variances of $\mathrm{RKF}_{0}$ are slightly larger than the ones of $\mathrm{RKF}_{1}$. In the second experiment, we apply these filters to the least favorable model (23) with $\tau=0$ and $c=10^{-1}$. The variances of $e_{t}^{1}$ and $e_{t}^{2}$ are depicted in the second row of Figure 2. Obviously, $\mathrm{RKF}_{0}$ is the best estimator because it has been designed with respect to this model. Although $\mathrm{RKF}_{1}$ has been designed with respect to another model, it performs better than KF. In the third experiment, we apply these filters to the least favorable model (23) with 

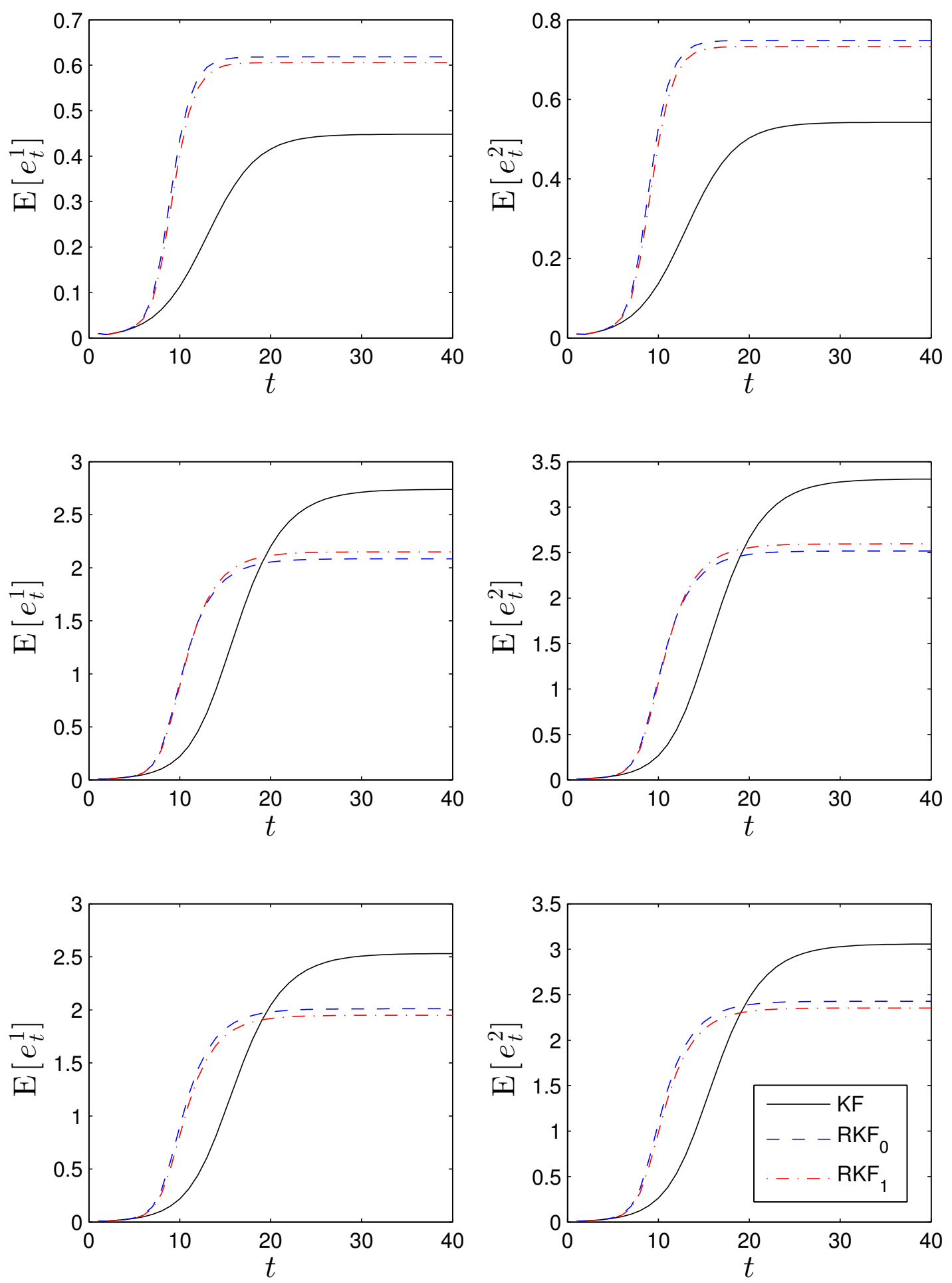

Fig. 2. Variances of $e_{t}^{1}$ and $e_{t}^{2}$ when the filters are applied to the nominal model (first row); to 23 with $\tau=0$ and $c=10^{-1}$ (second row); to 23) with $\tau=1$ and $c=10^{-1}$ (third row). Here $\mathrm{RKF}_{0}$ and $\mathrm{RKF}_{1}$ have $c=10^{-1}$. 
$\tau=1$ and $c=10^{-1}$. The variances of $e_{t}^{1}$ and $e_{t}^{2}$ are depicted in the third row of Figure 2. In this case $\mathrm{RKF}_{1}$ is the best estimator because it is optimal with respect to the underlying model. Also in this case, the worst estimator is KF. From these experiments we can conclude that:

- the smaller $\tau$ is, the more conservative the filter is, that is, the smaller $\tau$ is, the more the uncertainty class contains models with larger mean square error. This property has been noticed also for the static estimation problem in [19].

- the family of robust Kalman filters provide better performances than the standard Kalman filter, even in the case that the least favorable model belongs to an uncertainty class parametrized by a different $\tau$.

\section{B. Small tolerance}

We perform the same experiment three experiments of before where the unique difference is the tolerance which now is $c=5 \cdot 10^{-3}$, see Figure $3 . \mathrm{RKF}_{0}$ and $\mathrm{RKF}_{1}$ provides the same performance which is comparable with the one of KF. Therefore, as long as the discrepancy between the nominal and the least favorable model is not too large, then the performance of KF applied to 23) does not deteriorate too much.

\section{CONCLUSIONS}

In this paper, we have considered a robust filtering problem under incremental model perturbations characterized by the $\tau$-divergence family. The family of robust estimators we proposed is the solution to a minimax problem. These robust estimators have an iterative structure similar to the one of the Kalman filter. We have derived the corresponding least favorable models. Moreover, we have extended the risk sensitive filter to a family of risk sensitive like filters. Finally, a simulation study shows that parameter $\tau$ tunes how conservative the robust filter is.

\section{ACKNOWLEDGMENTS}

I am very grateful and indebted to Prof. Bernard C. Levy. During my visit at UC Davis in 2011, he has been always willing to clear up my doubts arising from his papers [8], [9]. 

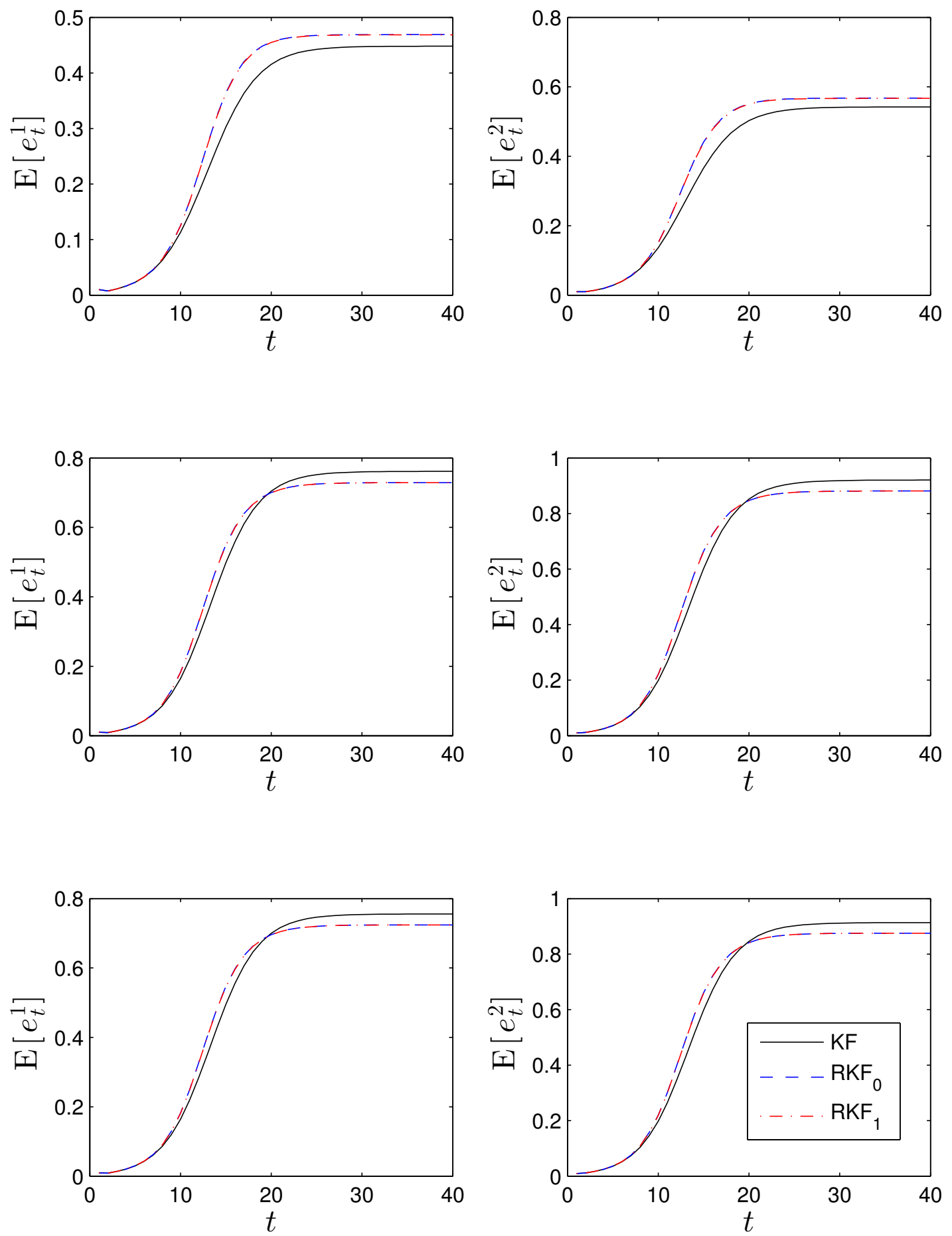

Fig. 3. Variances of $e_{t}^{1}$ and $e_{t}^{2}$ when the filters are applied to the nominal model (first row); to 23 with $\tau=0$ and $c=5 \cdot 10^{-3}$ (second row); to 23 with $\tau=1$ and $c=5 \cdot 10^{-3}$ (third row). Here $\mathrm{RKF}_{0}$ and $\mathrm{RKF}_{1}$ have $c=5 \cdot 10^{-3}$. 


\section{REFERENCES}

[1] R. Banavar and J. Speyer. Properties of risk-sensitive filters/estimators. IEEE Proc.-Control Theory Appl., 145(1), Jan. 1998.

[2] R. Boel, M. James, and I. Petersen. Robustness and risk-sensitive filtering. IEEE Trans. Automat. Control, 47(3):451-461, 2002.

[3] L. El Ghaoui and G. Calafiore. Robust filtering for discrete-time systems with bounded noise and parametric uncertainty. IEEE Trans. Automat. Control, 46(7):1084-1089, Jul. 2001.

[4] L. Hansen and T. Sargent. Robust estimation and control under commitment. Journal of Economic Theory, pages 2-258, 2005.

[5] L. Hansen and T. Sargent. Recursive robust estimation and control without commitment. Journal of Economic Theory, 136(1):1-27, 2007.

[6] L. Hansen and T. Sargent. Robustness. Princeton University Press, Princeton, NJ, 2008.

[7] B. Hassibi, A. Sayed, and T. Kailath. Indefinite-Quadratic Estimation and Control- A Unified Approach to $H^{2}$ and $H^{\infty}$ Theories. Soc. Indust. Appl. Math., Philadelphia, 1999.

[8] B. Levy and R. Nikoukhah. Robust least-squares estimation with a relative entropy constraint. Information Theory, IEEE Transactions on, 50(1):89-104, Jan. 2004.

[9] B. Levy and R. Nikoukhah. Robust state-space filtering under incremental model perturbations subject to a relative entropy tolerance. IEEE Trans. Automat. Control, 58:682-695, Mar. 2013.

[10] B. Levy and M. Zorzi. A contraction analysis of the convergence of risk-sensitive filters. SIAM J. Optimization Control, accepted, 2016.

[11] A. Sayed. A framework for state-space estimation with uncertain models. IEEE Trans. Automat. Control, 46(7):998-1013, Jul. 2001.

[12] J. Speyer and W. Chung. Stochastic Processes, Estimation, and Control. Advances in Design and Control. Soc. Indust. Applied Math., Philadelphia, 2008.

[13] J. Speyer, C. Fan, and R. Banavar. Optimal stochastic estimation with exponential cost criteria. In Proc. 31st IEEE Conf. Decision Control, pages 2293-2298, Tucson, AZ, Dec. 1992.

[14] P. Whittle. Risk-sensitive Optimal Control. J. Wiley, Chichester, England, 1980.

[15] M. Yoon, V. Ugrinovskii, and I. Petersen. Robust finite horizon minimax filtering for discrete-time stochastic uncertain systems. Syst. Control Lett., 52:99-112, 2004.

[16] M. Zorzi. A new family of high-resolution multivariate spectral estimators. IEEE Trans. Autom. Control, 59(4):892-904, Apr. 2014.

[17] M. Zorzi. Multivariate Spectral Estimation based on the concept of Optimal Prediction. IEEE Trans. Autom. Control, 60:1647-1652, Jun. 2014.

[18] M. Zorzi. Rational approximations of spectral densities based on the alpha divergence. Mathematics of Control, Signals, and Systems, 26(2):259-278, 2014.

[19] M. Zorzi. On the robustness of the Bayes and Winner estimators under model uncertainty. Submitted, 2015.

[20] M. Zorzi and B. Levy. On the convergence of a risk sensitive like filter. 54th IEEE Conference on Decision and Control $(C D C), 2015$.

[21] Mattia Zorzi. An interpretation of the dual problem of the THREE-like approaches. Automatica, 62:87-92, 2015. 